\title{
Research on Nintendo's Marketing Strategy During the Epidemic
}

\author{
Jialong $\mathrm{Liu}^{1}$ \\ ${ }^{1}$ ShiShi high school, Chengdu, Sichuan province, China, 641000 \\ *Corresponding author. Email: 3266219539@qq.com
}

\begin{abstract}
COVID-19 happened unexpectedly. The adverse impacts brought by COVID-19 are profound and continuing. For example, the revenues of firms in various fields are suffered from it. But there are still some surviving companies. So the marketing strategy of these companies is significant. This research studies a company called Nintendo that has a very good revenue during the epidemic. By using the Four Ps of marketing strategy theory, it analyzes the marketing strategy of Nintendo[1]. This paper will show the behavior of Nintendo from four aspects, product strategy, pricing strategy, placing strategy and promoting strategy. The product strategy points out that Nintendo designs its products based on the needs of customers, considering more for customers. This allows the buyers to feel more comfortable and tend to spend more in Nintendo. Second, the pricing strategy. Nintendo sets prices above not too much for their single one. Thus more people can afford their products, promoting them to purchase the products. Third, the placing strategy indicates that Nintendo minimizes their costs to maximize their profits and they always prepare a plan B. Therefore, they can avoid some independent troubles to some extent. Forth, the promoting strategy suggests that Nintendo establishes a nearly perfect after-sale system to guarantee the entertainment of the customers, reducing the decline of the number of customers.
\end{abstract}

Keywords: Nintendo, COVID-19, Marketing strategy, The Four Ps of Marketing

\section{INTRODUCTION}

The eruption of COVID-19 deeply influences the whole world. Everything becomes severe, making conditions more adverse for each field. Under this situation, a tremendous amount of firms was affected, many people lost their job, and a large scale of companies were forced to shut down. In addition, the original plans of other survival enterprises were also forced to alter. This study will utilize a strategy called the Four Ps of Marketing to analyze the marketing behaviors of Nintendo to figure out a better way to deal with the emergence of unexpected issues[1]. It can be predicted that there will be various and plentiful same difficult positions in the future. This research will provide companies with a suggestion to deal with unknown risks in the future.

\section{ANALYSIS ON THE MARKETING STRATEGY OF COVID-19}

Nintendo is a world-known entertainment product manufacturer in Japan, one of the three giants in the video game industry(the other two are Microsoft and SONY) and the pioneer of the modern video games industry. Nintendo was established on September 23, 1889[2]. The most famous games they made were the series of Zelda, Mario and Pokémon. In addition, their products are also well-known such as Switch, Wii, and Game Boy. In this case, it can be argued that Nintendo is an undoubtedly successful and solid firm.

\subsection{Product Strategy}

Product strategy refers to companies provide tangible and intangible products to satisfy consumer needs to achieve their marketing goals. Factors such as varieties, styles, and characteristics are the key to success.

\subsubsection{Varieties}

Switch has a cousin called Switch lite. It is a lowprofile version of switch. The main performances of Switch lite are as same as the Switch, but its price is less than Switch because of the lack of some additional functions and smaller size. 


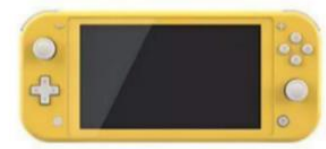

Switchlite

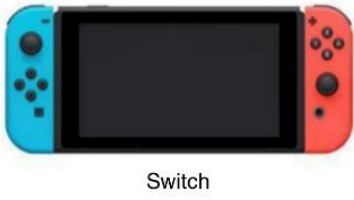

Figure 1. The appearance of Switch Lite and Switch

The size of Switch Lite is finer than Switch, so it will be more portable for people and its price will be less than Switch. Thus this can meet the need of workers who have to make several round trips from different places to different places, do not have the ability to afford Switch and just want to have fun by themselves. On the contrary, the Switch possesses more unique abilities, such as it allows multiple people to play together at the same time and its screen is larger, offering a more spectacular picture. These are suitable for those people who want to share fun with friends and want to experience a more profound impression.

\subsubsection{Styles}

The style of Switch is similar to the previous product, the PSP of SONY. However, there are slight differences, such as the monitor of Switch is larger than PSP and the gamepads of the Switch are longer than PSP, nearly rectangle. Moreover, the touch of Switch is more glossy than the touch of PSP.

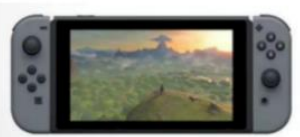

Switch

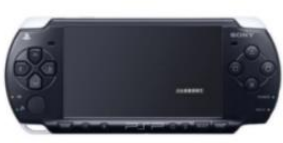

PSP

\subsubsection{Characteristics}

First, unlike other game consoles, Switch is born with a monitor. Thus, it enables people to use it everywhere.

Second, the primitive purpose of Switch is to allow every age of people can play it and share happiness with others because Nintendo has always insisted on opposing violence and pornography in the global game industry, and it is its mission to develop excellent games for all ages.

Third, the major innovation of Switch is the handles of Switch can be separated into two independent handles, while the additional handles of other brands' game consoles require customers to buy extra handles.

Fourth, this technology enables Switch to oversee the accuracy of the posture of people when they are playing somatosensory games like ring fit adventure and notice them, promoting them to do it more accurately[5]. As a result, they can be healthier.

\subsection{Pricing Strategy}

There are three decisive factors to determine the price: demand, cost, and competition. Plus, the price of a product should be between the interval of the cost of a product and the demand for a product.

Figure 2. The appearance of Switch and PSP

Nintendo Switch Sales(seruin)

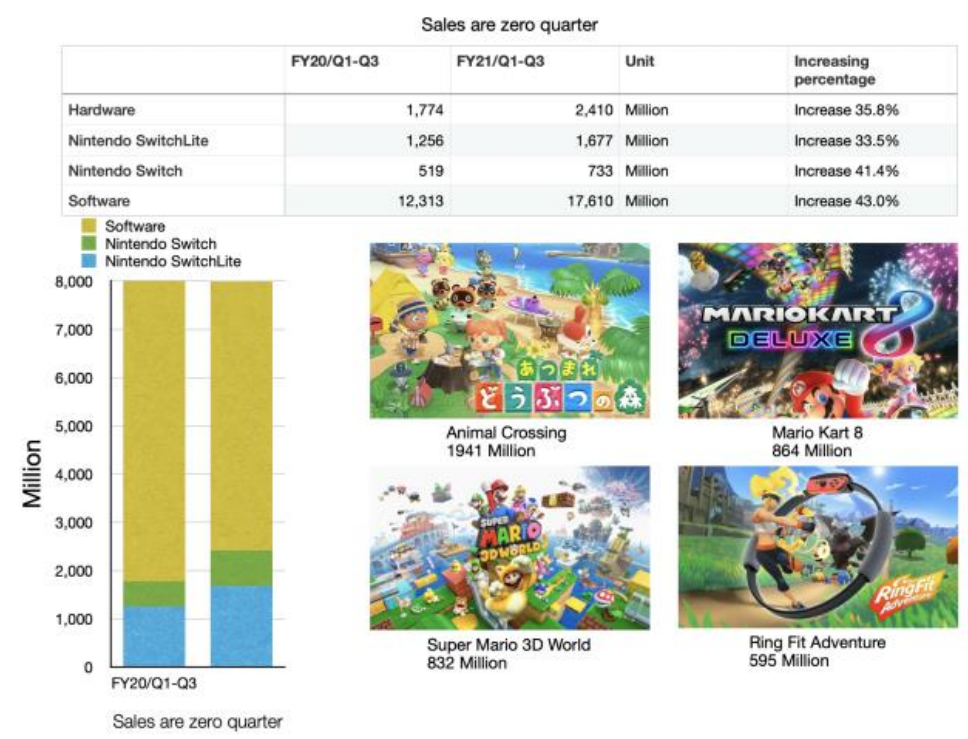

Figure 3. The sales of Nintendo Switch and Switch Lite 


\title{
Nintendo Switch family sell through
}

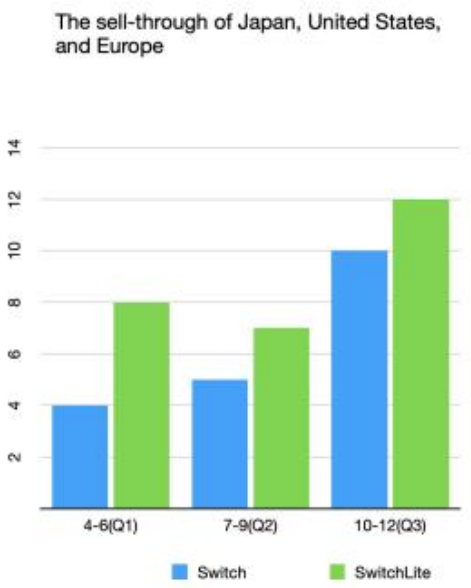
The third quarter 's performance was up from a year earlier, the largest since the launch.

\author{
At the end of the fourth holiday season, the
} global cumulative sell-through.

Over 74 Million

(Including Asia and Latin America, etc)

Figure 4. The sales of Nintendo Switch and Switch Lite

Table 1 and Table 2 show the demand for Switch in 2020[2]. Even there was a COVID-19, Nintendo still could find a way to restore their sales. Overall, the sales of Switch were increasing. However, when dividing the whole year into five parts, it can easily be found that when COVID-19 occupied Japan, Nintendo was unconscious about how to reduce their loss in the first period since the Japanese were afraid to COVID-19, isolating them in their home, causing there were fewer people cared about games market. However, things changed in the second period. According to the XiaoXiang morning news, during the epidemic, young volunteers took up nearly 530,000 people[3]. So people who were not volunteer had been at home for too long, they felt bored and agitated, and they vigorously needed entertainment. Comparing the data from the graph upon of Switch, Switch definitely fitted consumers as many people purchased it since it can permit two players to play each time by only buy one switch, while PS4 and XBOX One $\mathrm{X}$ requires extra gamepad which is around 64.99 dollars and 59.99 dollars each. Moreover, during this time, Nintendo published several games like Ring fits which costs 170 dollars, containing the game and the ring. These games with equipment allow people to keep their health without going to the gym, so they can exercise at the same time. In this case, Switch is a relatively worthy and ideal product for customers as it has an exercising function that is unique. Plus, games like Animal Crossing and Mario Kart 8 allow people to feel they are connected to each other, making them feel together, also promoting customers to buy switch, as only Switch can play these games. This stimulates the demand of switch to increases because the advantages of Switch completely match the desires of buyers, make more people aware of it, and its products are unique, which means they cannot be supported by other things like PS4 and XBOX One X. Thus even Nintendo did not regulate the price of Switch, it still creates profit for the company. A report that was published on April 5th, 2017 by a Japan Dismantling Company called Fomalhaut Techno revealed that the cost of a Switch was around 257 dollars, and the original price is 299.99 dollars. This means that the profits of each Switch are 42.99 dollars.

The main competitor of Switch is XBOX One X and PS4. The price of the XBOX One X and PS4 are higher than Switch, which is 399 dollars for XBOX One X and PS4. However, even the price of XBOX One X and PS4 is higher, the games can match the games players. When gamers are playing games, they are also socializing. On the contrary, Switch can only play the games of Nintendo, which means it can mate the Nintendo users. Furthermore, the picture quality produced by Switch is only 1080p, but the picture quality of XBOX One X and PS4 is $4 \mathrm{~K}$. The picture nether here shows that the images produced by XBOX One X and PS4 is obviously better than the image manufactured by Switch. In this case, Nintendo have to lower Switch's price to make up these two lacks.

Picture quality comparison: 

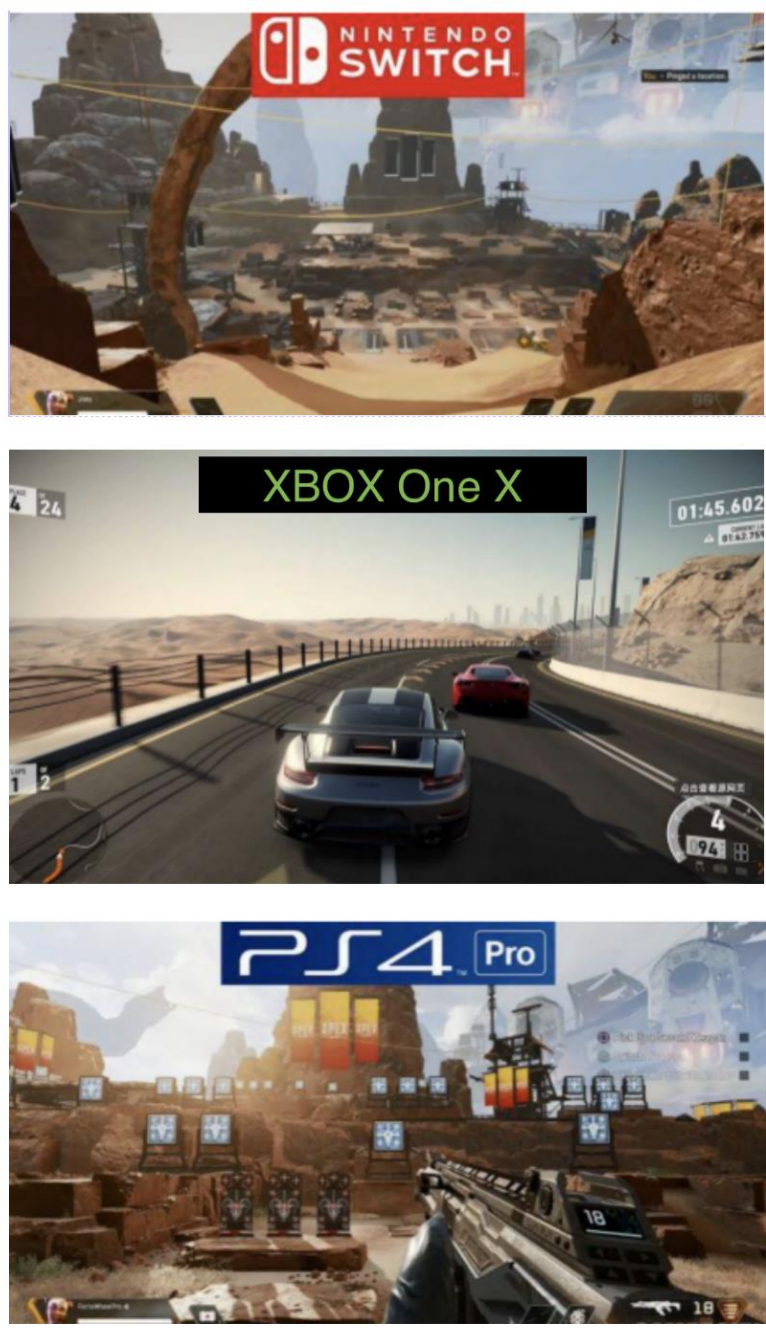

Figure 5. The image definition of Switch, PS4 and XBOX One X.

\subsection{Placing Strategy}

Placing strategy indicates the way that a product sold to customers from an enterprise. Nintendo has set several controlled companies in a different country. However, a special example is China. Since China has a severe policy, Nintendo cannot sell its products directly. Even it has avoided the restrictions of policy, it will face lots of problems when selling its software. Therefore, it has to discover a legal method. So they collaborate with a firm called Ique to enter the Chinese market. However, the efficiency of Ique is pretty low and it makes low quality of the Chinese editions of software. But Nintendo does not care about this since there are a tremendous amount of pirates, so they do not need to try so hard to create new games. Instead, they just have to release some latest information about the Chinese editions to stimulate buyers to buy them from official website or third-party offline stores.

On the contrary, COCID-19 actually did suffer this strategy by preventing customers to buy games because COVID-19 prevented the logistics and limited people at home. In this case, Nintendo initiated to lower the digital form of games to boost people to continue shopping for those who already had a Switch.

\subsection{Promoting Strategy}

Promoting strategy is used to spread the information to potential customers and stimulates them to purchase products. The two main promotion strategies of Nintendo are advertisements and the membership system.

First, the advertisements that are made by Nintendo are profound and creative[4]. They exhibit the advantages of their product straightly. Of course this is because of its natural advantages. Like the natural advantages of PS4 and $\mathrm{XBOX}$, One $\mathrm{X}$ is with picture quality, a multifunctional platform, and a limited edition commemorative edition of the host and software, which can duplicate the value and is more expensive.

Second, the membership system also assembles old player and new players, giving them a sense of honor and belonging[4]. And this will pave a way for the offspring of return customers to continue following Nintendo. Thus manufacture a solid base for the future of Nintendo.

The COVID-19 did not seem to influence the benefits that are produced by these two methods, thus Nintendo does not have to alter crucial changes. It seems to benefits the selling of Switch since people are occupied by their works and private issues, they have little time to release their pressure. However, COVID-19 trapped people at home. Thus they have more spare time for relaxing. But relaxing ways like traveling are impossible, so they can only do some things at home which will not be limited by place. Video games are regarded as the most suitable way to eliminate bored time. This eventually forced parts of them to pay attention to games. As a result, game console market and game market gain more opportunities. The outstanding advantages of Switch make it become the most popular console at that time. So more people purchased Switch and created more profits for Nintendo.

\section{CONCLUSION}

The Four Ps strategy possesses four parts, thus this paper will show four conclusions of each section. First, the product strategy shows that Nintendo designs its products to meet the needs of customers, considering more for customers. This allows their buyers to rely more on them and tend to choose Nintendo. Second, the pricing strategy suggests that Nintendo sets the prices properly. Thus more people can afford their products, promoting them to purchase the products. Third, the placing strategy shows that Nintendo minimizes the costs to maximize its profits. Also, they always possess a plan B. Therefore, they can avoid some independent troubles to some extent. Forth, the promoting strategy reflects that Nintendo builds a nearly perfect after-sale system to control the decreasing amounts of customers. 


\section{AUTHORS' CONTRIBUTIONS}

This paper is completed by the author independently.

\section{ACKNOWLEDGMENTS}

This paper would not have been possible without the consistent and valuable reference materials that I received from my supervisor, whose insightful guidance and enthusiastic encouragement in the course of my shaping this paper definitely gain my deepest gratitude.

\section{REFERENCES}

[1] Zhang Zhong, Ding Guo, Mei Yetian. The integrity and innovation of China's film industry in the postepidemic era[J]. Film Literature, 2021(04): 3-9.

[2] Li Xinyue. Analysis of Nintendo Switch's Marketing Strategy[J]. Manager, 2020, (05):74-75.

[3] Nintendo's 2020 earnings report released Ranger.com Sina.com 2021:3

[4] During the epidemic period, young volunteers took up nearly 530,000 person-times, with over $60 \%$ of post-90s and post-00s. 2020:12:18.

[5] Zhang Jucai, Qi Tian. Integrated marketing communication of Nintendo Switch game console[J]. Enterprise Research, 2018, No.501(06):26-30

[6] Stremersch S, Muller E, Peres R. Does new product growth accelerate across technology generations?[J]. Marketing Letters, 2010, 21(2):103-120. 